\title{
A CASE OF AMBLYOPIA DUE TO AMOEBIC DYSENTERY WITH COMPLETE RECOVERY
}

BY

\author{
ARnold Ferguson, F.R.C.S.(Edin.) \\ OPHTHALMIC AND AURAL SURGEON, JERSEY GENERAL HOSPITAL
}

J.W.S., aged 53 years, consulted me on January 16, 1923 for great deterioration of vision.

He had been in the Sudan and had contracted there dysentery and malaria. His life had been despaired of, and he showed evidences of this in his general condition. He had previously undergone emetine treatment. His visual acuity in each eye was 6/60. Skiascopy gave a correction in his right eye of $\frac{+1.00 \mathrm{D} \mathrm{Sph} .}{+0.25 \mathrm{D} \mathrm{Cyl} \text {. Ax. 105. }}$; in his lefteye of $\frac{+1.00 \mathrm{D} \mathrm{Sph} .}{+0.25 \text { D Cyl. Ax.V. }}$

No improvement with these glasses.

Discs pale, especially the right, so much so that it looked atrophic.

Referred to Col. Marett, the M.O.H. and Bacteriologist, for examination of stools. He reported the presence of cysts and suggested 1 grain emetine hypodermically daily with salines to keep the intestinal tract fluid. The point being that emetine kills the amoebae, but not the cysts. This is the reason why the double salt of emetine bismuthous iodide appears more efficient by producing a diarrhoea.

I did not see the patient again till October 10, when I found his vision to be $6 / 5$ in each eye with the correction.

At the same time his general condition had improved beyond recognition.

\section{NOTES ON MICROSCOPY OF THE LIVING EYE}

\section{Report of the Lang Clinical Research Scholarship, Royal London Ophthalmic Hospital}

\author{
BY \\ Basil Graves
}

Now, at a time when the clinical subject of microscopy of the living eye is arousing general interest, I desire to take the opportunity briefly to place on record the substance of certain observations which I have made in the course of a long time devoted to this work. When Senior Resident House Surgeon 
at Moorfields Hospital I interested myself in the subject of illumination of the eye, and as the outcome of observations made then in 1920 and 1921, I published a report on "Contact Illumination" (Brit. Jl. of Ophthal., May and August, 1921). I have since found no reason to call for alteration of the views expressed in that report with the exception that I would now, to some extent, modify certain opinions expressed with regard to the optical processes concerned. The usefulness of contact illumination is submerged by the slit-lamp and binocular microscope, save that in certain bedside cases, for whom the use of these is out of the question, it can still preserve a practical value. I then took up investigations with the slit-lamp and have pursued them ever since. During the period June, 1922, to October, 1923, I held the post of Lang Clinical Research Scholar at Moorfields Hospital. This scholarship terminated in October, 1923. I mention this because I desire to place on record that although I have since been pursuing research work of this nature, and shall continue to do so, the work will always be founded on the basis of earlier experience gained by the facilities afforded me at Moorfields Hospital whilst I held the posts to which I have referred. Some delay in rendering a report has been due to my desire to follow up certain of the cases concerned with a view to see how far their condition might undergo change with lapse of time.

This work has culminated in certain results. Because both of the difficulty of making and publishing numerous coloured illustrations, and of the endless variety of possible conditions which the slit-lamp and binocular microscope reveal in a manner clear to anyone familiar with the technique of their use, my object has always been not so much to describe numerous varieties of self-evident clinical conditions. I have aimed rather to ascertain the principles underlying various effects so that, by properly defining them, it would be possible to place the subject of clinical slit-lamp technique on a scientific basis which, when grasped, should enable observers to detect and describe fine clinical conditions, and to interpret them with an accuracy unimpaired by a want of knowledge of likely fallacies. I am sure that a proper understanding of such principles, which in themselves are not complicated, will soon come to be regarded by all ophthalmologists as being essential, and that none will regard the slit-lamp and binocular microscope as instruments to be used without deliberate precision.

Brief reference to the main methods of illumination and observation will be found in the Transactions of the Ophthalmological Society for 1923 ("Microscopy of the Living Eye"). The subject of, e.g. direct illumination, simple as it appears. 
is one on which much may be written which is of practical significance. One example of this would be the "epithelial impactline" referred to under "The Slit-lamp and the Histological Features of a Small Tumour at the Limbus," in the Transactions of the Ophthalmological Society for 1924. The subject of retroillumination is not one merely of vaguely focusing the light on the background of iris or lens, as may be realized from the description of the methods for detecting and observing a certain corneal disease which forms the subject of a report rendered to the Lang Clinical Research Committee. As I have brought forward in that report, whilst the cruder vacuolar changes within a lens or within a corneal epithelium are detectible by direct illumination in virtue of the specular reflexes and the shadows which they create, retro-illumination applied in a special way is necessary to detect the finest of such vacuoles; and unless the method of retroillumination is properly applied very fine pathological vacuolation in the lens, and in the interstices of the corneal epithelium, may readily pass undetected.

In certain cases of long-standing inflammation having crude conglomerate precipitated masses on the back of the cornea, the epithelium overlying the regions of endothelium on which these precipitates are deposited may reveal densely aggregated but fine intra-epithelial vacuolation; and this vacuolation may be strictly limited to these overlying regions of the epithelium and absent in regions of the epithelium which are not underlain by such precipitates. Now as these white precipitated masses can readily form a light optical background to the region of the epithelium lying in front of them, it might readily be contended that this statement is falsely based on an optical effect, and that the intraepithelial vacuolation, perhaps generally distributed, has been seen more readily over the keratic precipitates. Careful examination and interpretation of the optical effects in such cases has shown that the observation is correct, and that intra-epithelial vacuolation, having such definitely defined limits, can occur over the site of coarse keratic precipitates.

Examples of other features whose explanation is so dependent on optical principles are afforded by phenomena of the opticalsection of the cornea (Trans. Ophthal. Soc., 1924) and of the lens "Microscopy of the Normal Living Eye," British Medical Ássociation Opening Paper, Section of Ophthalmology, Annual Meeting, 1924) due to multiple lamination of structure. In the paper last referred to, which was one confined to appearances in the normal eye, a technique for readily observing the path of the beam through the normal aqueous fluid was described. The reason for thus emphasizing the possibility of seeing the beam in the normal aqueous fluid was that an observer who under- 
stands how, and is accustomed, to do this will be in a position to detect certain pathological variations in the aqueous fluid which would seem to have an important practical significance. This was only one of the aspects in which I was interested, and on which I began investigations, when I held the research appointment at Moorfields Hospital; and the results obtained then, and subsequently, have led to interesting conclusions with regard to changes in the aqueous fluid. One example of these to which I may allude here is that cases of simple retinal detachment come to exhibit a pathological increase in the visibility of the homogeneous flare or "outstanding beam" in the aqueous fluid.

I have found cases which, though having the aspect of being no more than cases of conjunctivitis, displayed the presence of intraocular disturbance by definite changes which were thus seen within the aqueous fluid.

I have long had some justification for supposing that, in the neighbourhood of certain ball-like precipitates on the back of the cornea, minute particles may be taken up into the cells of the endothelium on which these lie; the statement should, however, be made with reservation and perhaps not in the absence of a discussion of the reasons on which it is based.

When I was working at Moorfields Hospital certain appearances occasionally seen in acutely inflamed eyes led me to seek for the possibility of vascularization of the cornea taking place along the normal paths of the entering nerve-fibres. It is possible for an inexperienced observer to jump to this conclusion when no such condition exists, but subsequent clinical investigation has shown me that this process can take place. In interstitial keratitis it is possible-I do not say that it is common-for the corneal nerve-paths to be invaded by a very fine afferent-efferent vascular loop, and so precisely may the course taken by this vascularization be related to the nerves that, where the nerve divides dichotomously, the loop may extend along one or other, but not both, of the two branches before it reaches the terminal position which marks the sharp turning-point in the course of the tiny blood-stream. The decisive recognition of this clinical condition -at no time easy because of the accompanying photophobia-calls for an exact knowledge of precise technique.

Whilst speaking of interstitial keratitis one may refer to a defined and clear increase of the normal relucency of the posterior edge of the corneal beam often seen as a lasting sequel to a past attack. Mr. Treacher Collins has suggested to me that the condition may be the outcome of some changes on the part of the endothelium which represent an attempt, in the past, at protective opposition to the invasion of the cornea by spirochaetal toxic agents from the aqueous fluid. It is of interest to find this 
feature present in some of those supposedly uniocular cases whose sound eye, alleged never to have had an attack, reveals this condition as evidence of a past, unnoticed transient affection.

Early in slit-lamp work Koeppe's observation and deductions as to the liberation of pigment within the anterior chamber in cases of glaucoma led to controversy, Vogt affirming that the changes described by Koeppe were due to "physiological" causes only of a senile nature. I cannot but feel that there are certain cases of primary glaucoma in which the appearances of the atrophic iris suggest peculiar pigmentary changes, such for example, as the aggregation on the face of the stroma of collections of pigmentspecks which in colour are black rather than the more usual brown.

The appearance of lines suggestive of tension in some sector of the iris of certain cases of long-standing chronic primary glaucoma, where the iris-stroma rather than its ectodermal pigment-layers is atrophied, and the pupil is dilated, has led me to wonder if in these cases there has been atrophy, from nonactivity, of the sphincter-muscle, with subsequent persistence of function of a normally active or over-active dilatator muscle.

Accurate observations on the lens, as those on other regions, are dependent on a critically accurate technique. It does not seem to have been recognized that the lines of disjunction in the normal lens are capable of causing specular reflection in slight degree, and that their visibility. can hence vary slightly with the relationship of the angles made by the axes of illumination and observation with the normal to the curved faces which these lines define in the optical section. An interesting observation has been afforded by the detecting of an evenly defined accessory zone of disjunction in the lens of certain eyes which in the past have received concussion-injury.

The migration of the situation of pathological changes with growth of the lens-cortex lends itself to investigation with the slit-lamp. For example, in an eye which revealed fine particulate deposits throughout the corneal substance and changes about the anterior lens-capsule, both due to siderosis, a pyriform opacity in the centre of the anterior lens-capsule had its almost exact counterpart reduplicated at some depth from the surface; and spreading from the level of this reduplicated opacity, but lying at a regularly defined distance from the capsule, there was a zone which was defined, throughout almost the width of the lens, by the presence of fine siderotic punctate specks.

A series of observations made on the lens in cases of simple detachment of the retina suggests that the normal distance of separation of the capsular and discontinuity lines is prone to undergo increase in these cases. 
It might be thought that slit-lamp observations on the normal eye to detect variations in the curvature of the lens-capsules, under the influence of the accommodation-mechanism, would be easy; as a matter of fact it is for various reasons very difficult and unsatisfactory. But a case in which the two completely transparent and disunited lens-capsules were intact but for a tiny injury-hole in the anterior one, through which every vestige of lens-substance had long ago dissolved away, has afforded ample opportunity for direct observation, not only of the precise manner of relaxation of the capsules in the process of accommodation, but also of the influence on their state of tension produced by local reflexes or by various drugs instilled into the eye.

These instances which I have quoted are briefly cited examples of some of the observations made during much time devoted in the past three years to microscopy of the living eye. Slit-lamp effects resolve themselves largely into the simple question of illumination-contrasts. This is particularly seen, for example, in the detection of vacuolar spaces within relucent tissues. By reversing in a sense the principle of the slit-lamp, and working on a beam of darkness projected through illuminated tissue, I have obtained interesting results. I am much indebted to Professor O. Henker, Director of Messrs. Zeiss's Medical-Optical Department, for his trouble in the construction of experimental appliances which I hope may prove useful in this work.

I might here take the opportunity to mention that, unconnected with this work, I started at Moorfields Hospital, on another clinical investigation which led only to inconclusive results. It was based on this supposition: Patients suffering from acute following on chronic primary glaucoma, whose general history is carefully inquired into, can, I think, often be found to have been subject to long-standing symptoms many of which are justifiably referable in general to a heightened activity, or increased sensitiveness, of the sympathetic nervous system. It is also interesting to note in this connection that in women sympathetic activity is more predominant than in men, whilst vagus-activity is less so. It seemed rational, after these considerations, to see whether patients, who are doubtfully the subjects of primary glaucoma, exhibit a greater tendency towards dilatation of the pupil by the sympatho-mimetic drug p. hydroxyphenylethylamine or tyramine, than do normal persons. The observations were made by simply instilling the solution of the drug into the eye and not by subcutaneous injection; I cannot say that they led to conclusive or useful results. I wish to express my gratitude to Dr. Langdon Brown whose opinions, so kindly given, I sought on this matter. 